GLOSSARY

\title{
Cardiovascular epidemiology and prevention
}

\author{
F Rodríguez-Artalejo, J R Banegas Banegas
}

\section{This glossary contains a small number of terms from the field of cardiovascular disease, selected for a non-specialist readership.}

See end of article for authors' affiliations ....................

Correspondence to: Dr F Rodríguez-Artalejo, Departamento de Medicina Preventiva y Salud Pública, Facultad de Medicina, Universidad Autónoma de Madrid, Avda. Arzobispo Morcillo, sn, 28029 Madrid, Spain; fernando.artalejo@uam.es
$\mathrm{T}$ he scientific field of epidemiology and prevention of cardiovascular disease is enormous. Thus, in this glossary the reader will find only a comparatively small number of terms, selected according to what authors believe could be most interesting for a non-expert readership. To keep the list of terms to a minimum, we have decided not to define most of the well known cardiovascular risk factors, because they are becoming myriad. Lastly, we think this glossary could be of interest to a wide readership because it includes terms originally developed in the field of cardiovascular prevention, which were incorporated later to the field of "chronic disease" prevention.

\section{ANKLE-BRACHIAL INDEX (ABI)}

This is a simple, non-invasive test for quantifying the severity of arterial occlusion in the leg. It is performed by calculating the ratio of systolic blood pressure (using a Doppler device) in the humeral artery to the pressure reading in the dorsalis pedis and posterior tibial arteries. Diagnosis is confirmed if the ABI is $<0.9$ in either of the two legs, which shows that blood pressure is lower in the lower extremities than in the arm. A reduction in ABI is associated with generalised atherosclerotic disease and has been shown to predict cardiovascular mortality and morbidity in several populations.

\section{ARTERIOSCLEROSIS}

Thickening and hardening of the artery wall attributable to a number of causes, such as the presence of plaques (atheromas), age related loss of elastic fibres or calcification of the artery wall. Arteriosclerosis is associated with increased cardiovascular risk.

\section{ATHEROGENIC DYSLIPIDAEMIA}

A common lipid disorder characterised by three alterations, namely, raised triglyceride, high small low density lipoprotein cholesterol, and low high density lipoprotein (HDL) cholesterol. Usually the values of these three types of lipids are only slightly changed. Atherogenic dyslipidaemia is associated with obesity (particularly abdominal obesity), sedentary lifestyle, insulin resistance, type 2 diabetes mellitus, and premature coronary heart disease.
J Epidemiol Community Health 2004;58:1 16-119 ATHEROMATOSIS

A generalised vascular disorder affecting large and medium sized arteries, involving the existence of plaques (atheromas) under the arterial intima. These plaques are characterised by the abundance of cholesterol and inflammatory phenomena, such as the presence of monocytes and macrophages, fibroblasts, smooth muscle cells, and necrotic tissue. Dating from childhood and adolescence, atheromatosis is a lifelong progressive phenomenon that plays an essential part in the pathogeny of cardiovascular diseases. In many cases, the rupture of an atheroma, very rich in cholesterol and poor in fibrous tissue (unstable plaque), induces a thrombotic phenomenon, which leads in turn to rapid obstruction of the vessel lumen and acute cardiovascular event.

\section{ATHEROSCLEROSIS}

Arteriosclerosis attributable to presence of atheromas. Please see atheromatosis and arteriosclerosis.

\section{BARKER HYPOTHESIS}

Hypothesis formulated by D J P Barker, which postulates that a set of factors-principally nutritional deficiencies-that are active in the perinatal period programme metabolic changes that give rise to more increased cardiovascular risk factor values in early childhood and to a higher cardiovascular risk during adulthood. ${ }^{1}$ Some groundwork for this hypothesis is found in the study undertaken by A Forsdahl in Norway. ${ }^{2}$

\section{CARDIOVASCULAR RISK FACTOR}

Biological characteristic, conduct, or social variable that increases the likelihood of suffering or dying from cardiovascular disease. The term "risk factor" (RF) was first used in epidemiology by the Framingham study researchers in $1961 .^{3}$ RFs may have a causal or non-causal relation with cardiovascular disease. Among causal RFs are hypertension, smoking, hypercholesterolaemia, and diabetes mellitus. Although the mechanisms whereby these factors promote atherosclerosis or cardiovascular disease have not been totally elucidated, there is sufficient evidence to indicate that they play an independent causal part.

\section{CONDITIONAL RISK FACTOR}

Term used by S Grundy to refer to factors that are associated with a higher risk of cardiovascular disease but for which there is no definitive evidence of a causal role for two reasons: (1) their atherogenic potential may be small in comparison with that of the major risk factors; 
and (2) their frequency in the population may possibly not be sufficiently great to measure their independent effect in prospective studies. Such factors include increased serum concentrations of triglycerides, homocystein, blood coagulation factors (fibrinogen and plasminogen activator inhibitor-1) and lipoprotein (a). ${ }^{4}$

\section{FRAMINGHAM CARDIOVASCULAR EQUATIONS}

Set of equations, derived from the famous Framingham cohort study, which estimate mean cardiovascular risk for groups of subjects as a function of the magnitude of the main cardiovascular risk factors. The best known equations are those that score coronary risk and risk of stroke. The Framingham equations overestimate the risk in low risk, for example, Mediterranean populations. Calibration techniques have been developed, however, which use the distribution of risk factors and incidence of coronary events in such populations to improve the estimation of risk. The principal use of these equations is to select patients in a clinical setting to adjust the intensity of preventive interventions to individual subject's cardiovascular risk, thereby increasing the efficiency of primary prevention. The equations are also used to motivate patients to follow preventive recommendations.

\section{FRIEDEWALD FORMULA}

This formula is very widely used to estimate serum concentration of low density lipoprotein (LDL) cholesterol, by determining total cholesterol, HDL cholesterol, and triglycerides. The estimate is accurate where triglyceride concentrations are below $\leqslant 400 \mathrm{mg} / \mathrm{dl}$. The formula is as follows: LDL Cholesterol $=$ cholesterol total - HDL cholesterol-triglycerides $/ 5$ (all determinations in $\mathrm{mg} / \mathrm{dl}$ ). Determination of formula components requires the extraction of 9 to 12 hour fasting blood samples.

\section{INDIVIDUAL PREVENTION}

Prevention activities usually implemented through a clinical encounter between the subject and the health professional. Prevention is targeted at subjects with high cardiovascular risk, and the main instruments are screening for risk factors, advice to change lifestyles, and drug treatment. Individual prevention is complementary to population based prevention, and is aimed exclusively at subjects lying at the top end of the risk factor distribution for any given population.

\section{INSULIN RESISTANCE}

Also known as reduced insulin sensitivity, this consists of a deterioration in the degree of tissue response to the physiological action of insulin, probably because of a change in the structure of either insulin or its cell receptor, though the latter seem to be the predominant. Insulin resistance is manifested by hyperglucaemia in the presence of normal or even increased insulin levels in the blood. It is a generalised metabolic disorder associated with the so called metabolic syndrome, which is likewise known as insulin resistance syndrome. ${ }^{5}$

\section{KOROTKOF METHOD}

Auscultatory method of determining blood pressure, developed by the Russian scientist of the same name in 1905. The procedure includes the use of a von Recklinghausen stethoscope, Riva-Rocci cuff (both placed over the brachial artery), and Poiseuille mercury manometer. In contrast with earlier methods, this is non-invasive and comparatively simple, and has consequently become the standard technique for measuring blood pressure. This method relates the changes in sound detected by the stethoscope (Korotkof sounds) to successive blood pressure levels read on the manometer scale.
The first sound corresponds to systolic pressure, while the point before the disappearance of sounds (so called phase 5) corresponds to the diastolic pressure.

\section{LONG TERM PREVENTION}

Term used by the Adult Treatment Panel of the National Cholesterol Education Program to refer to lifetime primary prevention of cardiovascular disease, by impeding or delaying the development of premature or accelerated atherosclerosis. Generally, it is targeted at persons who are not at imminent risk of a cardiovascular event (usually younger subjects), and in essence consists of adopting a healthy lifestyle. ${ }^{6}$

\section{MAJOR RISK FACTOR}

These are factors that not only display the strongest association with cardiovascular disease but are also very common in the population. Hence, they account for an important proportion of cardiovascular disease cases in the population, and the potentially most effective prevention strategies are those targeted at their control. According to the American Heart Association, major cardiovascular risk factors are hypertension, smoking, hypercholesterolaemia, lack of physical activity, and obesity.

\section{METABOLIC SYNDROME}

Also known as syndrome $X$, this consists of the presence in a single subject of a group of cardiovascular risk factors of metabolic origin. These factors are abdominal obesity, atherogenic dyslipidaemia, arterial hypertension, insulin resistance often associated with glucose intolerance, and prothrombotic and proinflammatory states. In clinical practice, diagnosis is usually based on the presence of abdominal obesity (waist circumference $>102 \mathrm{~cm}$ in men and $>88 \mathrm{~cm}$ in women), raised plasma triglycerides $(\geqslant 150 \mathrm{mg} / \mathrm{dl})$, low plasma HDL cholesterol ( $<40 \mathrm{mg} / \mathrm{dl}$ in men and $<50 \mathrm{mg} / \mathrm{dl}$ in women), raised blood pressure ( $\geqslant 130 / 85 \mathrm{mg} / \mathrm{dl})$, and raised fasting glucaemia (110-125 mg/dl). Although the aetiology of the metabolic syndrome is not well known, it probably involves overweight/obesity, sedentary lifestyle, and genetic factors. Not only is metabolic syndrome a powerful cardiovascular risk factor but there is also evidence from clinical trials that control of its main components reduces cardiovascular risk. ${ }^{6}$

\section{MINNESOTA CODE}

Standardised procedure, commonly used in epidemiological studies, for reading and interpreting electrocardiograms.

\section{POPULATION BASED PREVENTION}

Also known as "mass prevention", this aims at producing a shift in the overall distribution of cardiovascular risk factors towards lower values. The main instruments used are legislation and health education/promotion, targeting the population as a whole. It is complementary to individual prevention and covers both high risk and moderate or low risk subjects. Population based prevention is undertaken to control those risk factors whose relation with the occurrence of cardiovascular disease is gradual and continuous (without threshold), and it could therefore be said that "the lower the risk factor, the better". This is the case of cholesterolaemia or blood pressure. This type of prevention has great potential for controlling cardiovascular disease in the population because most cases of disease occur among subjects who present with risk factors at values regarded by clinicians as "normal or only slightly elevated". ${ }^{7}$

\section{PREDISPOSING RISK FACTOR}

Term used by S Grundy to refer to distal factors in the causal chain of cardiovascular disease, factors such as obesity, 
sedentary lifestyle or male sex, which exert their action through intermediate, causal or conditional risk factors. ${ }^{4}$

\section{PRIMARY PREVENTION}

Set of activities aimed at reducing incidence of cardiovascular diseases through control of their risk factors. It could be carried out through activities targeted to individuals, populations, or both.

\section{PRIMORDIAL PREVENTION}

Term proposed to refer to the set of activities aimed at preventing or delaying the development or appearance of cardiovascular risk factors. $^{7}$

\section{PSYCHOSOCIAL RISK FACTOR}

A psychosocial factor may be defined as a measurement that potentially relates psychological phenomena to the social environment and to pathophysiological changes. ${ }^{8}$ There is evidence from prospective cohort studies showing that psychosocial factors, particularly depression, anxiety and social support, are independent aetiological and prognostic factors for coronary heart disease. There are several pathways that may serve to link psychosocial factors to coronary disease. Firstly, psychosocial factors may influence health behaviours (for example, smoking, diet or alcohol consumption) that are cardiovascular risk factors. Secondly, psychosocial factors may cause direct short or long term pathophysiological changes. Thirdly, psychosocial factors may affect access and provision of health care. ${ }^{8}$

\section{PULSE PRESSURE}

Difference between systolic and diastolic blood pressure. It is an important cardiovascular risk factor among middle age and older people.

\section{ROSE'S TEST}

Questionnaire designed to detect the presence of angina pectoris and widely used in cross sectional studies. Although it displays good sensitivity, its specificity is comparatively low. This questionnaire also enables intermittent claudication of the lower limbs to be identified. ${ }^{9}$

\section{SECONDARY PREVENTION}

Set of activities aimed at reducing the occurrence of cardiovascular events in subjects who have suffered a previous cardiovascular event. Generally this takes the form of appropriate treatment of acute events, control of risk factors, and cardiovascular rehabilitation. Accordingly, it differs from the concept of secondary prevention in the scheme drawn up by Leavell and Clark, in which secondary prevention refers to early disease detection, diagnosis, and treatment. The success of secondary cardiovascular prevention translates as a reduction in lethality, and it is regarded as an indirect quality indicator of cardiovascular health care.

\section{SHORT TERM PREVENTION}

Term used by the Adult Treatment Panel of the National Cholesterol Education Program to refer to the type of primary cardiovascular prevention aimed at preventing a coronary event in subjects who, in all likelihood, already have advanced atherosclerosis and present with a very high risk of a cardiovascular event in the short term. In this context, "short term" is generally taken to mean a period of 10 years or less, and coincides with the period of time to which coronary risk predictions based on the Framingham study equations are calculated. Prevention activities consist of lifestyle changes, to which drug treatment may be added. ${ }^{6}$

\section{SINGLE POPULATION THEORY}

This theory is based on the observation that population distribution of a number of cardiovascular risk factors is continuous and unimodal. This suggests that subjects exposed and not exposed to each risk factor belong to one and the same population, and that the determinants of exposure are thus not merely individual but rather population based. One deduction to be drawn from this theory is that an increase in the number of subjects with comparatively moderate risk factor values would be accompanied by an increase in the number of subjects with risk factor values at the top end of distribution. Evidence of this exists for alcohol and salt consumption, blood pressure and body weight, among other cardiovascular risk factors. The theory has important consequences for the design of cardiovascular risk factor control strategies. Hence, the promotion of moderate consumption of alcoholic beverages could have the undesirable effect of raising the numbers of those who consume these to excess; and yet a further example is the fact that the epidemic rise in obesity witnessed by many countries is also accompanied by weight gain among those having what is regarded as normal bodyweight. Consequently, the causes of a rise in obesity would affect not only obese subjects but the population as a whole, and, in addition to actions targeting obese subjects, control of obesity would call for actions targeting the entire population. The social mechanisms that explain the single population theory are not well elucidated.

\section{THE "THRIFTY" GENE HYPOTHESIS}

Theory proposed by J V Neel, based on the observation that, while diabetes mellitus hinders reproduction of the human species, it nevertheless seems to have been a common disorder for many centuries. To explain this paradox, it was postulated that the selective disadvantage of diabetes in itself was counteracted by a genetic mechanism that enabled energy to be conserved during periods of food scarcity, a phenomenon that would confer a selective advantage in the context of the living conditions during the greatest part of the mankind evolution. This advantage was attributed to what Neel termed a "thrifty" genotype, which subsequently became harmful in the living conditions of abundance enjoyed by modern societies. ${ }^{10}$ The thrifty gene hypothesis is one of the theories seeking to explain the rise in obesity and diabetes type 2 in some populations in the world. Not only is this theory based on archaeological and ethnographic data, but there is also evidence of a genetic influence in type 2 diabetes mellitus. However, the specific genes responsible for the "thrifty" genotype have not been identified.

\section{VASCULAR ENDOTHELIAL DYSFUNCTION}

There is an increasing recognition that atherosclerosis is an inflammatory disease that begins with dysfunction of the vascular endothelium. ${ }^{11}$ Endothelial dysfunction consists of an abnormality in the regulation of vascular tone and in antiplatelet activity by the endothelium. This term usually refers to a loss of the vasodilatation exerted by endothelial cells, mostly through the release of nitric oxide. In humans endothelial dysfunction is evaluated by pletismography. It is also reflected by increased plasma concentrations of soluble endothelial adhesion molecules. These molecules are raised in patients with all types of cardiovascular diseases as well as in patients who do not have clinically manifested cardiovascular disease CVD but who have coronary risk factors, such as smoking, hypertension, hypercholesterolaemia, hyperhomocysteinaemia, and diabetes mellitus. Drugs such as statins and dietary interventions by supplementation of omega-3 fatty acids, folic acid, and antioxidants have been shown to 
be effective in reducing systemic inflammation and reversing endothelial dysfunction.

\section{Authors' affiliations}

F Rodríguez-Artalejo, J R Banegas Banegas, Department of Preventive Medicine and Public Health, School of Medicine, Universidad Autónoma de Madrid, Spain

\section{REFERENCES}

1 Barker DJP. Mothers, babies and disease in later life. London: BMJ Books, 1994.

2 Forsdahl A. Are poor living conditions in childhood and adolescence an important risk factor for arteriosclerotic heart disease? Br J Prev Soc Med 1977;31:91-5

3 Kannel W, Dawber TR, Kagan A, et al. Factors of risk in the development of coronary heart disease-six year follow-up experience. Ann Intern Med 1961;55:33-50.
4 Grundy SM. Primary prevention of coronary heart disease. Integrating risk assessment with intervention. Circulation 1999;100:988-98.

5 Reaven GM. Banting lecture 1988. Role of insulin resistance in human disease. Diabetes 1988:37:1595-607.

6 Expert Panel on Detection, Evaluation, and Treatment of High Blood Cholesterol in Adults. Executive summary of the Third Report of The National Cholesterol Education Program (NCEP) Expert Panel on Detection, Evaluation, and Treatment of High Blood Cholesterol in Adult (Adult Treatment Panel III, or ATP III). JAMA 2001;285:2486-97.

7 Rose G. The strategy of preventive medicine. Oxford: Oxford University Press, 1992.

8 Hemingway H, Marmot M. Psychosocial factors in the aetiology and prognosis of coronary heart disease: systematic review of prospective cohort studies. BMJ 1999;318:1460-7.

9 Rose GA. The diagnosis of ischaemic heart pain and intermittent claudication in field surveys. Bull World Health Organ 1962;27:645-58.

10 Neel JV. Diabetes mellitus: a "thrifty" genotype rendered detrimental by "progress". Am J Hum Genet 1962;14:353-62.

11 Libby P. Changing concepts of atherogenesis. J Intern Med 2000;247:349-38.

Is it possible and worth keeping track of deaths within general practice? Results of a 15 year observational study

\section{B Beaumont, B Hurwitz}

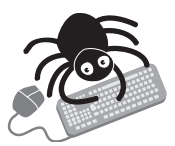

Please visit the Journal of Epidemiology and

Community Health website [www.jech. com] for a link to the full text of this article.
Aim: To assess the value of maintaining a death register in a general practice with particular reference to monitoring quality of care.

Design of study: Observational study.

Setting: Inner London general practice.

Method: The practice maintained a manual death register, retained medical records of all deceased patients, and requested information on cause of death from health authorities and coroners for 15 years.

Main outcome measures: Number and causes of deaths; 3 yearly age standardised death rates; proportion of deaths formally notified to the practice; place of death; source of cause of death information.

Results: During the study period 578 patients died. Practice age standardised death rates fell significantly from 35.59 to $27.12 / 1000.498(86.2 \%)$ deaths were formally notified to the practice, 392 within 7 days of death. Of 143 deaths reported to the coroner, only 45 coroners' reports were received. $360(64.1 \%)$ died in hospital, $139(24.8 \%)$ at home, and $38(6.8 \%)$ in a hospice. Death certificate cause of death information was obtained from patients' records in $33.6 \%(n=194)$ of cases and from health authority sources for $50 \%(n=289)$. The pattern of ascertained causes of deaths was similar to the national pattern.

Conclusion: A death register can examine trends in practice deaths by age and place of death and comparisons undertaken with nationally published mortality data. An accurate picture of cause of death cannot be generated from routine data flows alone. There is delay in informing GPs of patient deaths. Meaningful and timely monitoring of deaths cannot be undertaken by individual practices. National Statistics should provide routine analysis of GP death certificate information.

\ Quality and Safety in Health Care 2003;12:337-342. 